\title{
Acute Hematogenous Osteomyelitis in Children
}

\author{
Nimmy Thakolkaran, MBBS, ${ }^{1}$ Avinash K. Shetty, MD, ${ }^{2}$ \\ ${ }^{1}$ Department of Family Medicine, Mount Sinai Hospital, Chicago, IL ${ }^{2}$ Department of Pediatrics, Wake Forest School of Medicine, \\ Winston-Salem, NC
}

Background: The epidemiology of acute hematogenous osteomyelitis (AHO) in children has changed.

Methods: We reviewed the current literature regarding the epidemiology, microbiology, pathogenesis, clinical presentation, diagnosis, and antimicrobial management of AHO in children.

Results: Staphylococcus aureus is the most common microorganism causing pediatric AHO, followed by group A Streptococcus (GAS). AHO due to community-associated methicillin-resistant Staphylococcus aureus (MRSA) can cause severe and complicated disease. Pathogen isolation by culture is key for targeted antibiotic therapy. Polymerase chain reaction assay in tissue sample or joint fluid may enhance the yield of Kingella kingae. C-reactive protein is useful in diagnosis and monitoring the course of AHO. Magnetic resonance imaging is the preferred diagnostic imaging study for AHO. Clindamycin or vancomycin (for serious disease) is recommended for empiric therapy of suspected AHO due to MRSA depending on the geographic prevalence. Penicillinase-stable penicillins or first-generation cephalosporins are preferred antibiotics to treat methicillin-sensitive S aureus (MSSA) infection. Betalactam agents are the drugs of choice for treating AHO due to K kingae, GAS, or Streptococcus pneumoniae. For uncomplicated AHO due to MSSA, a short parenteral antibiotic course followed by oral therapy for a minimum total duration of 3-4 weeks is adequate. Complicated AHO due to MRSA may warrant prolonged therapy with surgical intervention.

Conclusion: Given the evolution of pathogens, the variability in clinical presentations and course ranging from simple to complex disease, and response to treatment, the management of AHO continues to evolve and warrants an individualized, multidisciplinary approach.

Keywords: Anti-bacterial agents, methicillin-resistant staphylococcus aureus, osteomyelitis

Address correspondence to Avinash K. Shetty, MD, Department of Pediatrics, Wake Forest School of Medicine, Medical Center Blvd., WinstonSalem, NC 27157. Tel: (336) 716-2740. Email: ashetty@wakehealth.edu

\section{INTRODUCTION}

Osteomyelitis is a bacterial infection of the bone associated with inflammation and bone destruction with an estimated incidence of approximately 8 per 100,000 children each year in high-income countries. ${ }^{1-2}$ Osteomyelitis can be classified as acute (duration of symptoms $<2$ weeks), subacute (duration of symptoms 2 weeks to 3 months), and chronic (long-standing infection that evolves over months to years). ${ }^{1}$ Osteomyelitis can result from direct inoculation from a penetrating trauma or can spread from a contiguous site of infection, but the most common mechanism of infection in children is hematogenous inoculation of the bone during an episode of bacteremia. ${ }^{3}$

\section{EPIDEMIOLOGY AND PATHOGENESIS}

Despite advances in diagnostic and treatment modalities, bone and joint infections are a major cause of morbidity and disease burden worldwide. Acute hematogenous osteomyelitis (AHO) is particularly common in children $<5$ years of age and typically affects the metaphysis because of the rich but slow blood flow of the growing bone. ${ }^{4}$ The microorganisms enter the bone via the nutrient artery and are lodged in the metaphyseal capillary loops where they begin to proliferate, resulting in the spread of inflammation. In neonates and children aged $<18$ months, the metaphyseal vessel loop and epiphyseal vessel are connected via transphyseal vessels traversing across the growth plate. Therefore, spread of metaphyseal infection to the epiphysis and joints can occur via transphyseal vessels. In older children, the vascular connections between the metaphysis and epiphyses are obliterated, and the two blood systems (metaphyseal loops and epiphyseal vessels) are separated from each other. Osteomyelitis of the proximal humerus or femur may also be associated with septic arthritis if the involved metaphysis is intracapsular. Boys are twice as likely to be affected than girls, and children aged $<5$ years accounted for more than $50 \%$ of cases of $\mathrm{AHO}^{5}$ Early diagnosis and appropriate treatment of osteomyelitis are imperative to avoid serious morbidity and permanent disability. ${ }^{4}$

\section{MICROBIOLOGY}

The bacterial etiology of osteomyelitis varies with age. ${ }^{5}$ The pathogen most often associated with AHO is Staphylococcus aureus in $80 \%$ of culture-positive cases, followed 
Table 1. Microorganisms Causing Osteomyelitis With Specific Risk Factors

\begin{tabular}{ll}
\hline Clinical Association/Risk Factor & Common Microorganism \\
\hline Sickle cell disease & Staphylococcus aureus, Salmonella spp, Streptococcus pneumoniae \\
Trauma & Polymicrobial (gram-negative, gram-positive, and anaerobic bacteria) \\
Chronic granulomatous disease & Serratia spp, Nocardia spp, Burkholderia cepacia, Aspergillus spp \\
Intravenous drug use & Staphylococcus aureus, Pseudomonas aeruginosa, Candida spp \\
Animal or human bites & Pasteurella multocida, Eikenella corrodens \\
Kitten exposure, HIV infection & Bartonella henselae \\
Exposure to farm animals & Coxiella burnetii \\
Diabetes & Polymicrobial (Staphylococcus aureus common, coagulase-negative Staphylococci, \\
Puncture wound & Streptococci, Enterobacteriaceae, and anaerobic bacteria) \\
\hline
\end{tabular}

HIV, human immunodeficiency virus.

by group A Streptococcus (GAS)..$^{1,5,6}$ In neonates, $S$ aureus, group B Streptococcus, and gram-negative enteric bacilli are usual pathogens. Neisseria gonorrhoeae must be considered in neonates and sexually active adolescents. ${ }^{5}$ In the child with sickle cell anemia, in addition to $S$ aureus, Salmonella spp frequently cause osteoarticular infections. ${ }^{5}$ In some countries (eg, Spain, France, United Kingdom, Israel, and Switzerland), Kingella kingae is being recognized increasingly as a common etiology of pediatric osteoarticular infections, especially in children $<5$ years. ${ }^{7}$ Data on the epidemiology of $K$ kingae infection in the United States are limited. In one US study of 99 children with septic arthritis, the diagnosis of $K$ kingae infection was made in 10 children aged $\leq 4$ years; polymerase chain reaction $(\mathrm{PCR})$ alone detected the pathogen in 8 cases. $^{8}$

Osteomyelitis caused by Haemophilus influenzae type b (Hib) is very rare in high-income countries since the widespread implementation of the vaccination program in the $1990 \mathrm{~s}^{9}$; historically, Hib accounted for $10 \%-15 \%$ of cases of osteomyelitis in unvaccinated children $<3$ years in low-income countries. ${ }^{5}$ Likewise, children who are not immunized or who are incompletely immunized against Streptococcus pneumoniae have a greater risk of developing invasive disease (eg, bacteremia, meningitis, pneumonia, and bone and joint infections). ${ }^{6}$

AHO caused by community-associated methicillinresistant $S$ aureus (CA-MRSA) has become common in many countries. ${ }^{4-6,9-11}$ The prevalence of MRSA varies significantly with geography. In one US study, MRSA was implicated in $30 \%-40 \%$ of pediatric osteoarticular infections. ${ }^{6}$ A 2016 study conducted at one large US institution reported that acute musculoskeletal infections caused by MRSA rose from 11.8\% in 2001-2002 to $34.8 \%$ in 2009-2010. ${ }^{11}$ In pediatric studies from Finland and Saudi Arabia, MRSA was not identified as an etiology, whereas CA-MRSA has emerged as a common pathogen causing skeletal infection in Romania and Greece. ${ }^{9,10}$

Most cases of $\mathrm{AHO}$ occur in children with no known risk factors. ${ }^{10}$ Table 1 depicts the various microorganisms causing osteomyelitis in patients with specific risk factors.

\section{CLINICAL PRESENTATION}

The clinical features of AHO vary with age and disease type. ${ }^{12} \mathrm{AHO}$ typically affects the metaphysis of long tubular bones, with approximately two-thirds of all cases involving the femur, tibia, or humerus. ${ }^{3-5}$ Although singlesite infection is most frequent, multifocal osteomyelitis can occur, especially in neonates and young infants. ${ }^{5,10}$ The onset of symptoms in $\mathrm{AHO}$ is often insidious. ${ }^{10}$ Children present with fever, localized pain, swelling, and rarely erythema around a long bone, limited range of motion, and limping or refusal to bear weight or use an extremity (pseudoparalysis). ${ }^{1,9}$ The most common clinical features of pediatric AHO reported in a 2012 systemic review are as follows: pain (81\%), localized signs/symptoms (70\%), fever $(62 \%)$, reduced range of movement $(50 \%)$, and reduced weight bearing (49\%). ${ }^{13}$ Other manifestations include fever of unknown origin and back pain in patients with vertebral osteomyelitis.

Systemic symptoms and signs such as high fever, tachycardia, and a painful limp are more commonly noted in children with MRSA osteomyelitis than in those with methicillinsensitive $S$ aureus (MSSA) osteomyelitis, although these findings are not specific only to MRSA. ${ }^{14}$ In contrast, children aged $<4$ years with $K$ kingae osteoarticular infection have a more benign presentation and course, with less than $15 \%$ febrile during admission and 39\% with normal C-reactive protein (CRP) levels. ${ }^{15}$

\section{DIFFERENTIAL DIAGNOSIS}

The differential diagnosis of AHO includes infection (eg, septic arthritis, cellulitis), trauma, malignancy (eg, osteoid osteoma, acute lymphoblastic leukemia, Ewing sarcoma, osteosarcoma), bone infarction (in children with sickle cell disease or other hemoglobinopathies), metabolic disease (eg, Gaucher disease), vitamin A deficiency, avascular necrosis, or chronic recurrent multifocal osteomyelitis. ${ }^{4,6,10}$

\section{DIAGNOSIS}

The diagnosis of $\mathrm{AHO}$ is established from a combination of history, physical examination, laboratory tests, imaging, and isolation of the microorganism from the bone, joint, or blood. ${ }^{9,10,16}$

\section{Inflammatory Markers}

The nonspecific markers of inflammation-CRP and erythrocyte sedimentation rate (ESR)-are commonly used in the initial evaluation of AHO. ${ }^{17} \mathrm{CRP}$ has a half-life of 19 hours 
and can also be followed to ensure the illness is resolving as expected; mean time to normalization of CRP is 7-10 days compared with ESR that is 2-3 weeks or more. ${ }^{10,17}$ Because CRP decreases more rapidly than ESR, in routine clinical practice, CRP is the preferred test to monitor the course and resolution of illness. ${ }^{17}$ The role of procalcitonin as a diagnostic aid in bone and joint infections is unclear. ${ }^{10}$ The white blood cell count may be normal in $\mathrm{AHO} .^{13}$

\section{Culture}

Detection of the causative organism and knowledge of antibiotic resistance patterns-especially for MRSA-are imperative for proper treatment of $\mathrm{AHO} .^{10,16}$ Thus, prior to administration of antibiotics, obtaining blood culture and bone biopsy/joint fluid samples is crucial whenever feasible. Bone/joint sample cultures have a higher diagnostic yield (40\%-50\% positive) compared to blood cultures (10\%$40 \%)$; an organism is recovered from any source in only approximately $40 \%-50 \%$ of cases of $\mathrm{AHO} .3,18$ Isolation of $\mathrm{K}$ kingae on standard culture media is difficult. Detection of $K$ kingae may be enhanced by direct inoculation of bony exudates or infected joint fluid into blood culture bottles, but the total reported detection rate (on solid media and blood culture vials) is approximately $33 \% .^{19}$

\section{Polymerase Chain Reaction}

PCR assays may enhance the yield of $K$ kingae. ${ }^{8,19}$ However, PCR technology is expensive and not readily available. Other newer diagnostic modalities include broad-range multiplex PCR/16S ribosomal RNA or DNA amplification to detect bacterial sequences for rapid diagnosis of bacterial etiology. ${ }^{20}$

\section{Imaging Techniques}

Plain radiography and ultrasound can be used to exclude other causes. A radiograph can exclude a fracture and malignancy (eg, Ewing sarcoma), while an ultrasound is very sensitive in detecting joint effusions. ${ }^{10}$ Lytic lesions and periosteal new bone formation may be evident on plain radiographs 2-3 weeks after onset of symptoms. ${ }^{1}$ Technetium radionuclide $\left({ }^{99 \mathrm{~m}} \mathrm{Tc}\right)$ bone scintigraphy is a sensitive test and useful to initially locate ill-defined sites of long bone infection or to detect multifocal disease, or it is used in situations when magnetic resonance imaging (MRI) is not feasible due to indwelling devices or braces. ${ }^{5}$ Radionuclide scans are frequently used as a diagnostic option in many countries but may yield false-negative results in infants. ${ }^{9,10}$ Computed tomography (CT) scan for diagnosis of bone and joint infection is generally not recommended in high-income countries because $\mathrm{CT}$ is less sensitive than $\mathrm{MRI}$ and involves exposure to high radiation doses. ${ }^{10}$

Currently, the imaging modality of choice is MRI, with significantly higher sensitivity (97\%-100\%) and specificity (92\%) compared to radiographs or bone scintigraphy (Figure). ${ }^{4,10}$ Early changes in osteomyelitis can be detected on MRI within 2-5 days of onset of disease. ${ }^{10} \mathrm{MRI}$ can also detect extraosseous manifestations or complications of $\mathrm{AHO}$ (such as pyomyositis, joint effusion, or subperiosteal abscess) and is invaluable for planning the surgical approach if required for management. ${ }^{9,10,21}$ The limitations of MRI are the cost, availability, long duration of scan time, and need for sedation or anesthesia. ${ }^{10,16}$

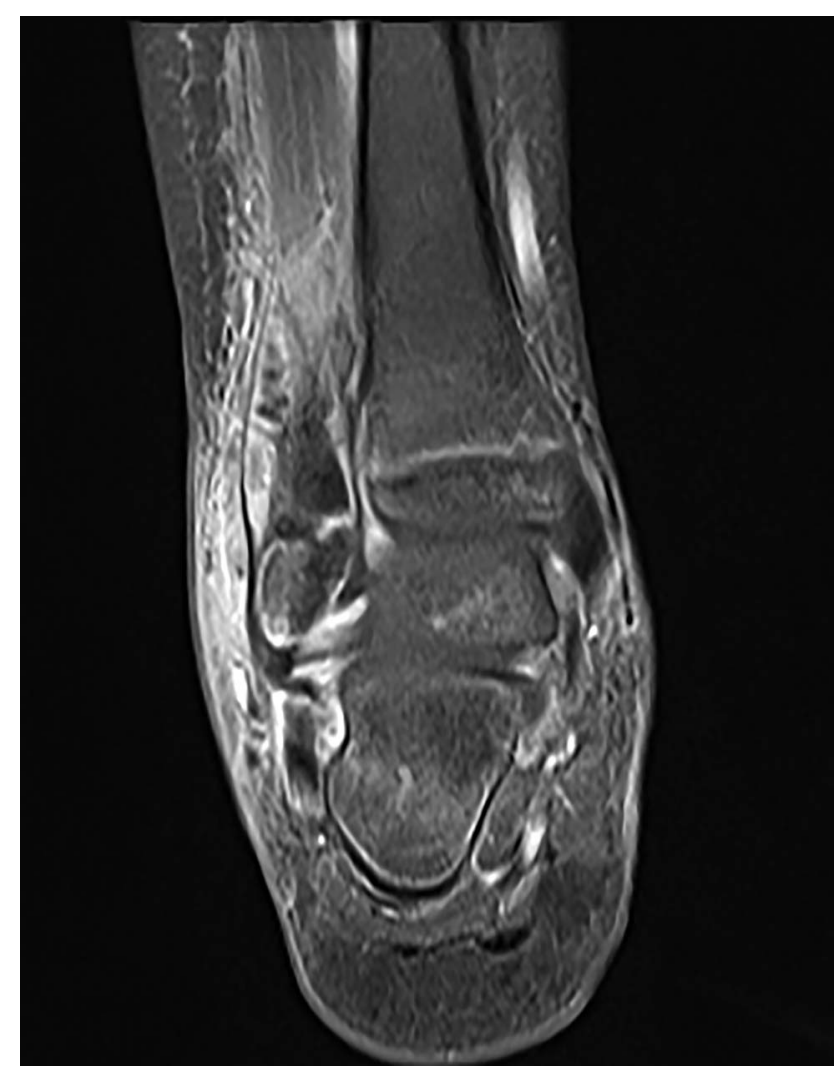

Figure. Magnetic resonance imaging of right ankle shows distal fibular osteomyelitis with subperiosteal abscess.

\section{MANAGEMENT}

The management of $\mathrm{AHO}$ in children is multidisciplinary, involving pediatricians, pediatric infectious disease specialists, orthopedic surgeons, and radiologists. ${ }^{10,12}$ The initial choice, length, and duration of antibiotic therapy depend on the causative organism, site of infection, associated complications, and clinical and laboratory response to treatment. $5,9,10,16$ In suspected cases of pediatric $\mathrm{AHO}$, empiric antibiotic therapy targeted against $S$ aureus (including CA-MRSA) must be initiated as soon as possible after appropriate specimens are collected for microbe isolation. ${ }^{1,5,10}$ In children $<4$ years with $\mathrm{AHO}$ receiving empiric antistaphylococcal therapy, consider adding a thirdgeneration cephalosporin to cover $K$ kingae.$^{5,9}$

\section{Antibiotic Therapy}

Clinicians must be aware of the local $S$ aureus susceptibility patterns to select the best agent for empiric therapy. 1,10,16,22 In geographic regions with prevalence of MSSA $>90 \%$, the initial empiric antimicrobial therapy for $\mathrm{AHO}$ beyond the neonatal period should include beta $(\beta)$-lactams, including an antistaphylococcal penicillin (eg, oxacillin or nafcillin) or first-generation cephalosporin (eg, cefazolin). ${ }^{1}$ Clindamycin is the preferred option if the patient is not critically ill in communities where the prevalence of MRSA is $>10 \%-15 \%$ and clindamycin resistance in MRSA isolates is $<10 \% .^{10,22}$ Vancomycin is recommended for empiric therapy if the prevalence of clindamycin-resistant $S$ aureus is $>10 \%-15 \%$ or if the patient has severe illness 
Table 2. Specific Pathogens and Antibiotic Treatment of Acute Osteomyelitis in Children

\begin{tabular}{|c|c|c|}
\hline Organism & Treatment & Alternatives \\
\hline Methicillin-susceptible Staphylococcus aureus & Antistaphylococcal penicillins ${ }^{a}$ & Cefazolin, clindamycin \\
\hline Methicillin-resistant Staphylococcus aureus & Clindamycin, ${ }^{\mathrm{b}}$ vancomycin & $\begin{array}{l}\text { Linezolid, daptomycin, } \\
\text { trimethoprim-sulfamethoxazole }\end{array}$ \\
\hline Group A Streptococcus & Penicillin G, ampicillin, amoxicillin & Clindamycin ${ }^{\mathrm{b}}$ \\
\hline \multirow[t]{3}{*}{ Streptococcus pneumoniae } & Ceftriaxone, ${ }^{\mathrm{b}}$ clindamycin $^{\mathrm{b}}$ & Vancomycin \\
\hline & Penicillin $\mathrm{G}^{\mathrm{b}}$ & \\
\hline & Ampicillin, amoxicillin ${ }^{b}$ & \\
\hline \multirow[t]{2}{*}{ Kingella kingae } & Ampicillin or ampicillin-sulbactam & \\
\hline & Cephalosporin & \\
\hline Enteric gram-negative rods & Ceftriaxone & Ciprofloxacin \\
\hline Pseudomonas aeruginosa & Ceftazidime, ticarcillin-clavulanate & Ciprofloxacin \\
\hline Neisseria gonorrhoeae & Ceftriaxone & Penicillin $G^{b}$ \\
\hline Salmonella spp & Ceftriaxone & Ampicillin ${ }^{b}$ \\
\hline Anaerobes & Penicillin, clindamycin & Metronidazole, meropenem \\
\hline
\end{tabular}

aOxacillin, nafcillin, cloxacillin, flucloxacillin, dicloxacillin.

${ }^{\mathrm{b}}$ For susceptible strains.

(such as endocarditis or deep venous thrombosis). ${ }^{5,10,16}$ Because the optimum penetration of vancomycin into bone is a concern, measurement of trough levels during therapy is prudent. ${ }^{1}$

Choice of empiric therapy also depends on the patient's immunization status and other risk factors such as sickle cell disease or immunodeficiency. In children who are not immunized or incompletely immunized during the first 5 years of life, in addition to $S$ aureus, empiric coverage for Hib is also warranted with cefuroxime or a combination of nafcillin or oxacillin or clindamycin with ceftriaxone. ${ }^{1}$ A thirdgeneration cephalosporin, such as cefotaxime or a fluoroquinolone, is indicated for patients with sickle cell disease to cover Salmonella spp in addition to antistaphylococcal coverage. $^{5}$

In neonatal osteomyelitis and septic arthritis, empiric treatment should be directed to $S$ aureus, group B Streptococci, and gram-negative bacteria, especially Escherichia coli. A penicillinase-stable penicillin (such as nafcillin or oxacillin) or vancomycin in combination with gentamicin or a third-generation cephalosporin (such as cefotaxime) provides excellent coverage for potential neonatal pathogens. ${ }^{5}$

Once the organism is isolated, subsequent antibiotic choice should be altered based on resistance profile. ${ }^{5} \beta$ lactam agents (oxacillin, nafcillin, and cefazolin) are the preferred agents if the isolated microorganism is MSSA. The $\beta$-lactam antibiotics have satisfactory bone penetration, proven efficacy for osteomyelitis, and an acceptable adverse effect profile when used in higher doses. ${ }^{22,23}$

Given its excellent oral bioavailability and bone penetration ability, clindamycin is a good choice for long-term oral treatment in osteomyelitis due to susceptible MRSA organisms without in vitro inducible resistance. ${ }^{24}$ Other alternative agents for MRSA osteomyelitis in selected circumstances include daptomycin and linezolid in patients who do not respond to vancomycin (Table 2). ${ }^{16,25}$ The role of trimethoprim-sulfamethoxazole for osteomyelitis due to
MRSA is anecdotal. ${ }^{25}$ Other agents (eg, glycolipopeptides, oxazolidinones, and ceftaroline, a novel fifth-generation cephalosporin with activity against MRSA) may be studied in future pediatric clinical trials. Addition of rifampin is recommended by some experts as part of combination therapy for complicated MRSA infection (such as bloodstream infections and device-related infections), but data on the benefit of rifampin in otherwise healthy children with $\mathrm{AHO}$ are lacking. ${ }^{10}$

Most $\beta$-lactam agents (such as ampicillin, ampicillinsulbactam, and cephalosporin) are effective against $K$ kingae, but the organism is not susceptible to vancomycin or clindamycin. ${ }^{5,9} \beta$-lactam antibiotics are also the drugs of choice for osteomyelitis due to GAS or $S$ pneumoniae. ${ }^{1}$

Culture-negative osteomyelitis is frequently encountered in clinical practice. In culture-negative cases of $\mathrm{AHO}$, the initial choice of empiric antimicrobial therapy should be continued in cases of documented clinical improvement with an associated decrease in CRP. Treatment with antistaphylococcal antibiotics is usually effective against culturenegative osteomyelitis; in addition, therapy against $K$ kingae should be considered in children $<4$ years. ${ }^{5,26}$

Transitioning From Intravenous to Oral Antibiotic Therapy. Despite the considerable variability in the management of pediatric $\mathrm{AHO}$, a growing body of evidence indicates that a shorter course of intravenous (IV) antibiotic therapy followed by an early transition to oral antibiotic therapy may offer a similar success rate in children with uncomplicated osteomyelitis compared to prolonged IV therapy alone while avoiding the complications related to the use of a venous catheter. $^{27-34}$

Studies have documented substantial risks associated with use of prolonged IV therapy for AHO. In a 2006 retrospective cohort study from the United States, of the 75 patients who received prolonged IV therapy ( $>2$ weeks) for $\mathrm{AHO}, 41 \%$ had $\geq 1$ central venous catheter (CVC)associated complication. CVC malfunction or displacement 
Table 3. Oral Antibiotic Dosages for Treating Acute Hematogenous Osteomyelitis in Children ${ }^{5,37}$

\begin{tabular}{lcc}
\hline Antibiotic & Dose, $\mathbf{m g} / \mathbf{k g} / \mathbf{d a y}$ & Doses/day \\
\hline Clindamycin & $30-40$ & $3-4$ \\
Cephalexin & 100 & 4 \\
Dicloxacillin & $75-100$ & 4 \\
Amoxicillin & 100 & 4 \\
\hline
\end{tabular}

occurred in 17 (23\%) patients, catheter-associated bloodstream infection occurred in $8(11 \%)$, fever with negative blood culture results was noted in $8(11 \%)$, and local skin infection at the site of catheter insertion occurred in $4(5 \%)$ patients. ${ }^{35}$

In another report, children with $\mathrm{AHO}$ sent home on IV therapy were 2.1 times more likely to experience catheter-related adverse events compared with children on oral antibiotics only. ${ }^{36}$ The study suggested that institutional culture and tradition rather than patient characteristics were driving therapeutic choices regarding prolonged IV therapy. ${ }^{36}$ Oral antibiotic therapy is less expensive and more convenient than IV therapy, and it can be instituted as stepdown treatment when the patient's clinical status has improved (eg, resolution of fever and pain), when bacteremia resolves (if initially positive), when CRP level declines to $<3 \mathrm{mg} / \mathrm{dL}$, and when compliance with therapy and follow-up is excellent. ${ }^{10,37,38}$

A randomized controlled trial conducted in Finland showed that most cases of childhood $\mathrm{AHO}$ can be treated with 2-4 days of IV antibiotics followed by 20 or 30 days of oral antibiotics (large doses of clindamycin or a first-generation cephalosporin) in patients who respond quickly and whose CRP values normalize within 10 days. ${ }^{32}$ In this study, all cases of osteomyelitis were caused by MSSA. Therefore, the therapeutic approach proposed by the authors may not be applicable to settings where MRSA osteomyelitis is common. Two additional trials (conducted in Chile and Australia/United Kingdom) that included predominantly MSSA cases also demonstrated no difference in outcomes when the duration of IV antibiotic therapy was $<1$ week. ${ }^{33,34}$ A systematic review from the United Kingdom published in 2013 also concluded that uncomplicated $\mathrm{AHO}$ in children $>3$ months should be treated with 3-4 days of IV antibiotics followed by the transition to oral antibiotics if the child shows a good clinical response. ${ }^{39}$ Using higher doses of oral $\beta$-lactam antibiotics is crucial to ensure adequate antibiotics levels in the bloodstream (Table 3). ${ }^{5,37}$

Duration of Antibiotic Therapy. Limited evidence exists on the initial antibiotic choice or the optimal length of parenteral or oral treatment of pediatric $\mathrm{AHO}{ }^{39}$ The appropriate duration of therapy for $\mathrm{AHO}$ is unclear and depends on the infecting pathogen, severity of the disease, concomitant septic joint, and the host. ${ }^{9,40}$ Historic data indicate high rates of relapse if the duration of antibiotic therapy is $<3$ weeks. ${ }^{41}$ However, this study was limited by a retrospective study design, and the reported treatment failures may also be related to other factors such as delay in surgical drainage of subperiosteal or bone abscess in some patients that resulted in chronic disease and subsequent relapse and no surgical intervention in half of the patients diagnosed with chronic osteomyelitis at presentation. ${ }^{41}$ Data from a clinical trial from
Finland published in 2010 support a shorter duration of therapy (20 days) compared to 30 days for uncomplicated $\mathrm{AHO}$, especially if the disease is caused by MSSA. ${ }^{32}$ A systematic review published in 2013 also recommended a shorter duration of parenteral antibiotic therapy (3-4 days) followed by oral antibiotic therapy to a total duration of 3 weeks in children with uncomplicated $\mathrm{AHO}$ (Grade 2 recommendation). ${ }^{39}$ However, a longer duration of therapy (including prolonged parenteral course) ranging from 4-6 weeks may be warranted for a complicated clinical course (eg, sepsis, venous thrombosis), resistant or virulent microorganism (such as MRSA, Panton-Valentine leukocidin [PVL]-positive $S$ aureus), and pelvic or spinal column involvement. ${ }^{10,16,40,42,43}$ In contrast to MRSA osteomyelitis, multidrug-resistant pneumococcal strains causing $\mathrm{AHO}$ and septic arthritis are not associated with an increased risk of complicated disease. ${ }^{44}$ Data are also lacking on the effectiveness of shorter durations of therapy in selected populations with $\mathrm{AHO}$, such as neonates, immunocompromised or malnourished children, and children with sickle cell disease. ${ }^{39}$

\section{Surgical Intervention}

Studies have shown that appropriate antibiotic therapy alone without surgical intervention may suffice for $90 \%$ of cases of $\mathrm{AHO}$. $^{9,13,32}$ In some cases of complicated $\mathrm{AHO}$ due to CA-MRSA, surgical incision and drainage (including multiple procedures) may be indicated. ${ }^{40}$ The indications for surgical management in $\mathrm{AHO}$ include persistent symptoms (fever, local inflammation) not responding to empiric antibiotic therapy, the presence of periosteal or other deep soft tissue abscess (more common with MRSA or strains expressing virulence genes such as PVL), concomitant septic arthritis especially of the hip and shoulder joint, presence of necrotic bone, and formation of a sinus tract. ${ }^{13,40,42}$

\section{COMPLICATIONS}

AHO due to MRSA may be associated with more complicated disease compared with $\mathrm{AHO}$ caused by MSSA or organisms other than $S$ aureus, including subperiosteal and intraosseous abscesses, deep vein thrombosis at a site adjacent to the infected bone, septic pulmonary emboli, disseminated infection with multiorgan failure, pathologic fractures, longer median hospital stay, increased requirement of surgical intervention to drain deep abscesses, and even chronic sequelae..$^{10,11,40,43,45}$ Severe disease could be related to an $S$ aureus virulence factor, PVL, a cytotoxin that destroys leucocytes, that has been found to be more prevalent in MRSA. $5,10,42,46-48$ A delay in the diagnosis and initiation of appropriate treatment can lead to potentially devastating morbidity, including sepsis, chronic infection, disruption of longitudinal bone growth, and angular deformity. ${ }^{13}$ Pelvic osteomyelitis is often associated with pyomyositis and may be related to infection with CA-MRSA or PVL production. ${ }^{49,50}$ Close follow-up within 2 weeks following discharge is recommended for patients with $\mathrm{AHO}$ to ensure continued clinical improvement. 51

\section{CONCLUSION}

Pediatric $\mathrm{AHO}$ is a serious infection worldwide. Given the evolution of pathogens, variability in clinical presentations and course ranging from simple to complex disease, and response to treatment, the management of $\mathrm{AHO}$ continues 
to evolve and warrants an individualized, multidisciplinary approach. With the emergence of CA-MRSA in some countries, early diagnosis, identification of the pathogen, and appropriate antibiotic use are crucial to achieve favorable outcomes and avoid complications.

The diagnosis of $\mathrm{AHO}$ is made using a combination of factors including clinical findings, inflammatory markers, and MRI and obtaining a specimen (bone and blood) for culture when feasible. Thereafter, empiric antibiotic therapy based on epidemiology and resistance pattern of the etiologic agent for the patient's specific geographic area must be started. For simple, uncomplicated infections, a short course of IV antibiotics followed by oral antibiotics is acceptable if clinical status improves and CRP declines. However, for complicated $\mathrm{AHO}$ (eg, multifocal disease, abscesses, venous thrombosis) due to resistant bacteria such as MRSA, prolonged parenteral therapy in conjunction with surgical intervention as appropriate may be necessary.

To achieve optimal outcomes, antibiotic treatment should be continued until the CRP concentration has normalized or significantly decreased. Sequential determinations of CRP are important to monitor response to treatment in $\mathrm{AHO}$ patients infected with MSSA and for patients with $\mathrm{AHO}$ caused by organisms other than $S$ aureus.

\section{ACKNOWLEDGMENTS}

The authors have no financial or proprietary interest in the subject matter of this article.

\section{REFERENCES}

1. Peltola $\mathrm{H}$, Pääkkönen M. Acute osteomyelitis in children. N Engl J Med. 2014 Jan 23;370(4):4:352-360. doi: 10.1056/NEJMra1213956.

2. Riise ØR, Kirkhus E, Handeland KS, et al. Childhood osteomyelitis-incidence and differentiation from other acute onset musculoskeletal features in a population-based study. BMC Pediatr. 2008 Oct 20;8:45. doi: 10.1186/1471-2431-8-45.

3. Krogstad P. Osteomyelitis. In: Cherry J, Demmler-Harrison GJ, Kaplan SL, Steinbach WJ, Hotez PJ, eds. Feigin and Cherry's Textbook of Pediatric Infectious Diseases. 7th ed. Philadelphia, PA: Elsevier Saunders; 2014:711-727.

4. Iliadis $A D$, Ramachandran M. Paediatric bone and joint infections. EFORT Open Rev. 2017 Mar 13;2(1):7-12. doi: 10.1302/2058-5241.2.160027.

5. Hong DK, Gutierrez K. Osteomyelitis. In: Long SS, Prober CG, Fischer M, eds. Principles and Practice of Pediatric Infectious Diseases. 5th ed. Philadelphia, PA: Elsevier; 2018:480-486.

6. Arnold SR, Elias D, Buckingham SC, et al. Changing patterns of acute hematogenous osteomyelitis and septic arthritis: emergence of community-associated methicillin-resistant Staphylococcus aureus. J Pediatr Orthop. 2006 Nov-Dec;26(6):703-708.

7. Weiss-Salz I, Yagupsky P. Kingella kingae infections in children: an update. In: Curtis N, Finn A, Pollard AJ, eds. Hot Topics in Infection and Immunity in Children VIII: Advances in Experimental Medicine and Biology, Vol 719. New York, NY: Springer; 2012:67-80.

8. Carter K, Doern C, Jo CH, Copley LA. The clinical usefulness of polymerase chain reaction as a supplemental diagnostic tool in the evaluation and the treatment of children with septic arthritis. J Pediatr Orthop. 2016 Mar;36(2):167-172. doi: 10.1097/BPO.0000000000000411.
9. Dodwell ER. Osteomyelitis and septic arthritis in children: current concepts. Curr Opin Pediatr. 2013 Feb;25(1):58-63. doi: 10.1097/MOP.0b013e32835c2b42.

10. Saavedra-Lozano J, Falup-Pecurariu O, Faust SN, et al. Bone and joint infections. Pediatr Infect Dis J. 2017 Aug;36(8):788-799. doi: 10.1097/INF.0000000000001635.

11. Sarkissian EJ, Gans I, Gunderson MA, Myers SH, Spiegel DA, Flynn JM. Community-acquired methicillin-resistant Staphylococcus aureus musculoskeletal infections: emerging trends over the past decade. J Pediatr Orthop. 2016 Apr-May;36(3):323-327. doi: 10.1097/BPO.0000000000000439.

12. Faust SN, Clark J, Pallett A, Clarke NM. Managing bone and joint infection in children. Arch Dis Child. 2012 Jun;97(6):545-553. doi: 10.1136/archdischild-2011-301089.

13. Dartnell J, Ramachandran M, Katchburian M. Haematogenous acute and subacute paediatric osteomyelitis: a systemic review of the literature. J Bone Joint Surg Br. 2012 May;94(5):584-595. doi: 10.1302/0301-620X.94B5.28523.

14. Ju KL, Zurakowski D, Kocher MS. Differentiating between methicillin-resistant and methicillin-sensitive Staphylococcus aureus osteomyelitis in children: an evidence-based clinical prediction algorithm. J Bone Joint Surg Am. 2011 Sep;93(18):1693-1701. doi: 10.2106/JBJS.J.01154.

15. Ceroni D, Cherkaoui A, Ferey S, Kaelin A, Schrenzel J. Kingella kingae osteoarticular infections in young children: clinical features and contribution of a new specific real-time PCR assay to the diagnosis. J Pediatr Orthop. 2010 Apr-May;30(3):301-304. doi: 10.1097/BPO.0b013e3181d4732f.

16. Liu C, Bayer A, Cosgrove SE, et al; Infectious Diseases Society of America. Clinical practice guidelines by the Infectious Diseases Society of America for the treatment of methicillin-resistant Staphylococcus aureus infections in adults and children. Clin Infect Dis. 2011 Feb 1;52(3):e18-e55. doi: 10.1093/cid/ciq146.

17. Pääkkönen $M$, Kallio $M J$, Kallio $P E$, Peltola H. Sensitivity of erythrocyte sedimentation rate and C-reactive protein in childhood bone and joint infections. Clin Orthop Relat Res. 2010 Mar;468(3):861-866. doi: 10.1007/s11999-009-0936-1.

18. Moumile K, Merckx J, Glorion C, Pouliquen JC, Berche P, Ferroni A. Bacterial etiology of acute osteoarticular infections in children. Acta Paediatr. 2005 Apr;94(4):419-422.

19. Yagupsky P, Porsch E, St Geme JW 3rd. Kingela kingae: an emerging pathogen in young children. Pediatrics. 2011 Mar;127(3):557-565. doi: 10.1542/peds.2010-1867.

20. Fenollar F, Lévy PY, Raoult D. Usefulness of broad-range PCR for the diagnosis of osteoarticular infections. Curr Opin Rheumatol. 2008 Jul;20(4):463-470. doi: 10.1097/BOR.0b013e3283032030.

21. Browne LP, Mason EO, Kaplan SL. Optimal imaging strategy for community-acquired Staphylococcus aureus musculoskeletal infections in children. Pediatr Radiol. 2008 Aug;38(8):841-847. doi: 10.1007/s00247-008-0888-8.

22. Peltola H, Pääkkönen M, Kallio P, Kallio MJ; OM-SA Study Group. Clindamycin vs. first-generation cephalosporins for acute osteoarticular infections of childhood-a prospective quasi-randomized controlled trial. Clin Microbiol Infect. 2012 Jun;18(6):582-589. doi: 10.1111/j.1469-0691.2011.03643.x.

23. Tetzlaff TR, Howard JB, McCraken GH, Calderon E, Larrondo J. Antibiotic concentrations in pus and bone of children with osteomyelitis. J Pediatr. 1978 Jan;92(1):135-140.

24. Feigin RD, Pickering LK, Anderson D, Keeney RE, Shackleford PG. Clindamycin treatment of osteomyelitis and septic arthritis in children. Pediatrics. 1975 Feb;55(2):213-223.

25. Arnold JC, Bradley JS. Osteoarticular infections in children. Infect Dis Clin North Am. 2015 Sep;29(3):557-574. doi: 10.1016/j.idc.2015.05.012. 
26. Floyed RL, Steele RW. Culture-negative osteomyelitis. Pediatr Infect Dis J. 2003 Aug;22(8):731-736.

27. Grimbly C, Odenbach J, Vandermeer B, Forgie S, Curtis S. Parenteral and oral antibiotic duration for treatment of pediatric osteomyelitis: a systemic review protocol. Syst Rev. 2013 Oct 7;2:92. doi: 10.1186/2046-4053-2-92.

28. Karen R, Shan SS, Srivastava R, et al; Pediatric Research in Inpatient Settings Network. Comparative effectiveness of intravenous $v$ oral antibiotics for acute osteomyelitis in children. JAMA Pediatr. 2015 Feb;169(2):120-128. doi: 10.1001/jamapediatrics.2014.2822.

29. Zaoutis T, Localio AR, Leckerman K, Saddlemire S, Bertoch D, Keren R. Prolonged intravenous therapy versus early transition to oral antimicrobial therapy for acute osteomyelitis in children. Pediatrics. 2009 Feb;123(2):636-642. doi: 10.1542/peds.2008-0596.

30. Pääkkönen M, Kallio MJ, Kallio PE, Peltola H. Shortened hospital stay for childhood bone and joint infections: analysis of 265 prospectively collected culture-positive cases in 1983-2005. Scand J Infect Dis. 2012 Sep;44(9):683-688. doi: 10.3109/00365548.2012.673729.

31. Peltola $H$, Pääkkönen $M$, Kallio $P$, Kallio $M J$; Osteomyelitis-Septic Arthritis(OM-SA) Study Group. Prospective, randomized trial of 10 days versus 30 days of antimicrobial treatment, including a short-term course of parenteral therapy, for childhood septic arthritis. Clin Infect Dis. 2009 May 1;48(9):1201-1210. doi: 10.1086/597582.

32. Peltola $H$, Pääkkönen $M$, Kallio $P$, Kallio $M J$; Osteomyelitis-Septic Arthritis Study Group. Short-versus long-term antimicrobial treatment for acute hematogenous osteomyelitis of childhood: prospective, randomized trial on 131 culture-positive cases. Pediatr Infect Dis J. 2010 Dec;29(12):1123-1128. doi: 10.1097/INF.0b013e3181f55a89.

33. Prado S MA, Lizama C M, Peña D A, Valenzuela M C, Viviani S T. Short duration of initial intravenous treatment in 70 pediatric patients with osteoarticular infections [in Spanish]. Rev Chilena Infectol. 2008 Feb;25(1):30-36. doi: 10.4067/S0716-10182008000100007.

34. Jagodzinski NA, Kanwar R, Graham K, Bache CE. Prospective evaluation of a shortened regimen of treatment for acute osteomyelitis and septic arthritis in children. J Pediatr Orthop. 2009 Jul-Aug;29(5):518-525. doi: 10.1097/BPO.0b013e3181ab472d.

35. Ruebner R, Keren R, Coffin S, Chu J, Horn D, Zaoutis TE. Complications of central venous catheters used for the treatment of acute hematogenous osteomyelitis. Pediatrics. 2006 Apr;117(4):1210-1215.

36. Wheeler AM, Heizer HR, Todd JK. Influence of culture results on management and outcome of pediatric osteomyelitis and/or septic arthritis. J Pediatric Infect Dis Soc. 2012 Jun;1(2):152-156. doi: $10.1093 /$ jpids/pis035.

37. Nelson JD. A critical review of the role of oral antibiotics in the management of hematogenous topics in infectious disease. In: Remington RS, Swartz MN, eds. Current Clinical Topics in Infectious Disease. Hoboken, NJ: Wiley-Blackwell; 1996:64-74.
38. Arnold JC, Cannavino CR, Ross MK, et al. Acute bacterial osteoarticular infections: eight-year analysis of C-reactive protein for oral step-down therapy. Pediatrics. 2012 Oct;130(4):e821-e828. doi: 10.1542/peds.2012-0220.

39. Howard-Jones AR, Isaacs D. Systematic review of duration and choice of systemic antibiotic therapy for acute haematogenous bacterial osteomyelitis in children. J Paediatr Child Health. 2013 Sep;49(9):760-768. doi: 10.1111/jpc.12251.

40. Kaplan SL. Recent lessons for the management of bone and joint infections. J Infect. 2014 Jan;68 Suppl 1:51-56. doi: 10.1016/j.jinf.2013.09.014.

41. Dich VQ, Nelson JD, Haltalin KC. Osteomyelitis in infants and children. A review of 163 cases. Am J Dis Child. 1975 Nov;129(11):1273-1278.

42. Dohin B, Gillet $Y$, Kohler R, et al. Pediatric bone and joint infections caused by Panton-Valentine leukocidin-positive Staphylococcus aureus. Pediatr Infect Dis J. 2007 Nov;26(11):1042-1048.

43. Crary SE, Buchannan GR, Drake CE, Journeycake JM. Venous thrombosis and thromboembolism in children with osteomyelitis. J Pediatr. 2006 Oct;149(4):537-541.

44. Bradley JS, Kaplan SL, Tan TQ, et al. Pediatric pneumococcal bone and joint infections. The Pediatric Multicenter Pneumococcal Surveillance Study Group (PMPSSG). Pediatrics. 1998 Dec;102(6):1376-1382.

45. Belthur MV, Birchansky SB, Verdugo AA, et al. Pathologic fractures in children with acute Staphylococcus aureus osteomyelitis. J Bone Joint Surg Am. 2012 Jan 4;94(1):34-42. doi: 10.2106/JBJS.J.01915.

46. Bocchini CE, Hulten KG, Mason EO Jr, Gonzalez BE, Hammerman WA, Kaplan SL. Panton-Valentine leukocidin genes are associated with enhanced inflammatory response and local disease in acute hematogenous Staphylococcus aureus osteomyelitis in children. Pediatrics. 2006 Feb;117(2):433-440.

47. Shallcross LJ, Fragaszy E, Johnson AM, Hayward AC. The role of the Panton-Valentine leucocidin toxin in staphylococcal disease: a systematic review and meta-analysis. Lancet Infect Dis. 2013 Jan;13(1):43-54. doi: 10.1016/S1473-3099(12)70238-4.

48. Martínez-Aguilar G, Avalos-Mishaan A, Hulten $\mathrm{K}$, Hammerman W, Mason EOJr, Kaplan SL. Community-acquired, methicillin-resistant and methicillin-susceptible Staphylococcus aureus musculoskeletal infections in children. Pediatr Infect Dis J. 2004 Aug;23(8):701-706.

49. Pannaraj PS, Hulten KG, Gonzalez BE, Mason EO Jr, Kaplan SL. Infective pyomyositis and myositis in children in the era of community-acquired methicillin-resistant Staphylococcus aureus infection. Clin Infect Dis. 2006 Oct 15;43(8):953-960.

50. Moriaty $P$, Leung C, Walsh M, Nourse C. Increasing pyomyositis presentations among children in Queensland, Australia. Pediatr Infect Dis J. 2015 Jan;34(1):1-4. doi: 10.1097/INF.0000000000000470.

51. Wood JB, Johnson DP. Prolonged intravenous instead of oral antibiotics for acute hematogenous osteomyelitis in children. J Hosp Med. 2016 Jul; 11 (7):505-508. doi: 10.1002/jhm.2549.

This article meets the Accreditation Council for Graduate Medical Education and the American Board of Medical Specialties Maintenance of Certification competencies for Patient Care, Medical Knowledge, and Practice-Based Learning and Improvement. 